\title{
A novel method of subxiphoid video-assisted thoracic surgery for thymectomy
}

\author{
Lei Gao ${ }^{1,2 \#}$, Jieming Lu ${ }^{1 \#}$, Zhimin Shen ${ }^{1 \#}$, Hongbo Chen ${ }^{1}$, Mingqiang Kang ${ }^{1,2,3}$ \\ ${ }^{1}$ Department of Thoracic Surgery, Fujian Medical University Union Hospital, Fuzhou, China; ${ }^{2}$ Key Laboratory of Ministry of Education for \\ Gastrointestinal Cancer, Fujian Medical University, Fuzhou, China; ${ }^{3}$ Fujian Key Laboratory of Cardio-Thoracic Surgery, Fujian Medical University, \\ Fuzhou, China \\ Contributions: (I) Conception and design: M Kang, L Gao; (II) Administrative support: L Gao; (III) Provision of study materials or patients: J Lu; \\ (IV) Collection and assembly of data: J Lu, H Chen; (V) Data analysis and interpretation: Z Shen; (VI) Manuscript writing: All authors; (VII) Final \\ approval of manuscript: All authors. \\ \#These authors contributed equally to this work. \\ Correspondence to: Professor Mingqiang Kang; Doctor Lei Gao. Department of Thoracic Surgery, Fujian Medical University Union Hospital, 29 \\ Xinquan Road, Gulou, Fuzhou 350001, China. Email: mingqiang_kang@126.com; 15980200120@163.com.
}

Background: With advances in thoracoscopic surgical instruments and techniques, subxiphoid videoassisted thoracic surgery (S-VATS) has become the main approach for anterior mediastinal tumor resection under thoracoscopy. However, the drawbacks of S-VATS, including it being a relatively unfixed surgical procedure, make it complicated and difficult for unexperienced surgeons to master.

Methods: This study retrospectively reviewed and analyzed consecutive patients with anterior mediastinal tumor or myasthenia gravis (MG) who underwent S-VATS at the Fujian Medical University Union Hospital, China, between March 2015 and April 2019.Patients were divided into the conventional group and the "four-zone one-way" group. Intraoperative and postoperative variables were compared between the groups. Cumulative sum (CUSUM) analysis was applied to determine the operation time (OT)-learning curve of the S-VATS "four-zone one-way" method.

Results: A total of 82 patients were included in this analysis, of which, 40 patients underwent the conventional method of S-VATS and 42 patients underwent the "four-zone one-way" method. Patients in the "four-zone one-way" group had significantly shorter OT $(138.50 \pm 29.43$ and $118.00 \pm 28.18$ minutes, respectively; $\mathrm{P}=0.002)$ and significantly less blood loss $(36.00 \pm 20.16$ and $23.92 \pm 14.96 \mathrm{~mL}$, respectively; $\mathrm{P}=0.003$ ) compared with patients in the conventional group. Our data indicated that there was no difference of the efficacy of MG treatment between the 2 groups. The difference in the preoperative and postoperative quantitative MG scoring system score (QMG-score) and the dose reduction of cholinesterase inhibitors was comparable between patients in the 2 groups. According to the CUSUM analysis curve, after a steady improvement over phase I (cases 1-12 for the traditional method and cases 1-5 for the "four-zone one-way" method), the surgical procedure could be mastered. Phase III occurred after case 26 in the traditional group and case 28 in the "four-zone one-way" group, and is characterized by rapid improvements.

Conclusions: Compared with the conventional method of S-VATS, the "four-zone one-way" method significantly decreased OT and estimated blood loss. These results demonstrated the feasibility and safety of the "four-zone one-way" method of S-VATS.

Keywords: Subxiphoid approach; thymectomy; thoracic surgery

Submitted Jun 25, 2021. Accepted for publication Aug 20, 2021.

doi: 10.21037/atm-21-4070

View this article at: https://dx.doi.org/10.21037/atm-21-4070 


\section{Introduction}

Complete resection for malignant mediastinal tumors is important for achieving satisfactory long-term survival. Median sternotomy is an effective approach (1), however, the associated significant trauma means that patients suffer many postoperative complications and recovery is slow. With advances in thoracoscopic surgical instruments and techniques, the use of video-assisted thoracic surgery (VATS) has been shown to be safe and capable of achieving oncological results similar to those of open surgery $(2,3)$. Apart from the intercostal thoracic approach, there are several minimally invasive approaches including the subxiphoid approach and robotic techniques $(4,5)$. Since it was firstly proposed in 1999 by Kido et al. (4), subxiphoid video-assisted thoracic surgery (S-VATS) has become the main approach for anterior mediastinal tumor resection under thoracoscopic surgery. Compared with the intercostal thoracic approach, S-VATS provides a better operative view (6) and reduces the operative time, blood loss, postoperative hospital stay, and postoperative pain $(7,8)$.

However, S-VATS is an unfixed and complicated surgical procedure (9). This, together with its other drawbacks, make it difficult for unexperienced surgeons to master this technique . With the experience and understanding of S-VATS in our department, the subxiphoid surgical method was improved and the "four-zone one-way" method was pioneered for anterior mediastinal tumor resections. The "four-zone one-way" method divides the intact thymus gland and its surrounding tissues into four zones by the anterior median line of the sternum and the lower pole of the left innominate vein and therefore optimizes the surgical procedure by dissecting each zone one by one in a single direction (clockwise or counterclockwise). Based on previous literature, no clinical studies have been found regarding this surgical approach. As a scoring system based on quantitative testing of sentinel muscle groups, the quantitative MG scoring system score (QMGscore) was used to access the differences between preoperative and postoperative symptoms in our study. This report explored the feasibility and safety of the "four-zone one-way" method of S-VATS and compared it with the traditional method.

We present the following article in accordance with the STROBE reporting (available at https://dx.doi.org/10.21037/ atm-21-4070).

\section{Methods}

\section{Study design}

An open cohort design was implemented. This study retrospectively reviewed and analyzed consecutive patients with anterior mediastinal mass or myasthenia gravis (MG) who underwent S-VATS in our hospital between March 2015 and April 2019. Between March 2015 and May 2017, patients underwent the conventional method of S-VATS and were assigned to the conventional group. From May 2017, as the subxiphoid surgical methods were improved, the "four-zone one-way" method was implemented. Subsequently in June 2017, all patients who underwent the "four-zone one-way" method of S-VATS were assigned to the "four-zone one-way" group. All operations were performed by the same surgical team at the Union Hospital Affiliated to Fujian Medical University, China. All procedures performed in this study involving human participants were in accordance with the Declaration of Helsinki (as revised in 2013). The study was approved by institutional ethics board of Fujian Medical University [No.: 2021 Association Ethics Examination No. (030)] and informed consent was taken from all the patients.

\section{Patients}

Patients were enrolled in the study if the following inclusion criteria were satisfied: (I) imaging data showed anterior mediastinal tumor with a tendency of enlargement of anterior mediastinal tumor during follow-up, or discomfort such as chest tightness and shortness of breath; (II) chest computed tomography (CT) scan and enhancement indicated that the anterior mediastinal tumor had clear boundaries with the peripheral nerves and large vessels and organs, and there was no bilateral lung, mediastinal lymph node, or distant metastasis; (III) retrosternal goiter; (IV) in cases where the anterior mediastinal tumor was complicated with MG, the MG symptoms were well controlled; (V) not complicated with primary malignant tumors in other sites; (VI) preoperative electrocardiograph (ECG), heart color ultrasound, lung function, and other examinations indicated good cardiopulmonary function and patients could tolerate surgical treatment; (VII) blood tests [including routine blood, coagulation function, liver and kidney function, eight surveys before blood transfusion, blood group identification and antibody screening, beta human chorionic gonadotropin ( $\beta-\mathrm{HCG}$ ), and alpha-fetoprotein $(\mathrm{AFP})]$ and routine stool tests (including routine stool and $\mathrm{OB}$ tests and routine urine tests) showed no abnormalities; and (VIII) patients and their family members fully understood the surgical plan, were willing to participate in this study, and provided written informed consent. The 
following exclusion criteria were applied: (I) chest CT scan and enhancement indicated anterior mediastinal tumor, adhesion with surrounding tissues, nerves, organs and large vessels, unclear boundary, or multiple metastasis in bilateral lungs and other sites; (II) patients with heart, lung, or other important organ dysfunction, and patients who were determined to be unsuitable for surgery during the anesthetic evaluation; (III) patients with a previous history of thymic tumor resection and consideration of recurrence and metastasis, or patients undergoing open-chest cardiac surgery; (IV) patients with MG who were receiving medical treatment, and who had been determined to be unsuitable for surgery after evaluation; (V) intraoperative endoscopic exploration suggested that the tumor could not be completely resected and turned to thoracotomy, or the pleural pericardium and other extensive metastatic patients only underwent thoracoscopic biopsy; (VI) a history of other malignant tumors, and the possibility of anterior mediastinal metastasis was considered; and (VII) patients with serious mental illness.

\section{Surgical techniques}

\section{The conventional S-VATS method}

After general anesthesia, the patient was placed in the supine position with legs spread apart. The surgeon was located between the legs of the patient, with one assistant located on the left side of the patient, and the other assistant located on the right side of the patient.

A $2 \mathrm{~cm}$ transverse incision was made at two transverse fingers below the xiphoid process, and a blunt separation was made with the index finger to the posterior sternum to establish a tunnel to the anterior mediastinum. A Trocar with a diameter of $10 \mathrm{~mm}$ was placed and connected to a pneumoperitoneum tube to establish artificial pneumothorax. The pressure of the $\mathrm{CO}_{2}$ gas was adjusted to $6-8 \mathrm{kPa}$, and the anterior mediastinum was fully exposed. The posterior sternal space was confirmed by thoracoscopy. A $1 \mathrm{~cm}$ longitudinal incision was then made at $1 \mathrm{~cm}$ below the intersection of the midclavicular line and costal arch on both sides as an auxiliary operation hole, and a $10 \mathrm{~mm}$ Trocar was placed under endoscopic vision (Figure 1A).

To expose the posterior sternum (Figure 1B), an ultrasonic knife was used to gradually separate the posterior sternum space from bottom to top. During the anterior mediastinal exploration, the location, size, boundary, capsule integrity of the anterior mediastinal tumor and its relationship with the left innominate vein and superior vena cava were determined.

To separate the tumor, the anterior boundary of the mediastinal tumor was gradually separated from bottom to top along the posterior sternum with an ultrasonic knife to the thoracic entrance. The lower boundary of the tumor was carefully dissected and separated along the lower edge of the mediastinal tumor with an ultrasonic knife. The left and right mediastinal pleura were opened with an ultrasound knife along the phrenic nerve, and important vessels such as the left sub clavicular artery, the left common carotid artery, the left innominate vein, superior vena cava, right innominate vein, and front edge of brachiocephalic vein were dissected carefully, so as to completely dissociate the left and left posterior bounds of the mediastinal tumor. The scope of dissection of the peri thymus adipose tissue included the bilateral anterior mediastinum, the diaphragmatic angle, and the peri thymus adipose tissue. The fat was removed completely, together with the anterior mediastinal tumor, and the specimens were placed into the specimen extractor through the operating hole and dragged out. After complete hemostasis, the wound was covered with hemostasis material and sprayed with biological glue preparation. One posterior sternal drainage tube was indwelling. After gauze and instruments were counted, the incision was sutured layer by layer.

\section{The "four-zone one-way" S-VATS method}

Following the procedures described above for the S-VATS traditional method, the patient was anesthetized and incisions were made to allow for anterior mediastinal exploration.

To separate the tumor, the "four-zone one-way" method was used instead of the conventional method. The anterior boundary of the mediastinal tumor was gradually separated from bottom to top along the posterior sternum with an ultrasonic knife to the thoracic entrance. The lower boundary of the tumor was carefully dissected and separated along the lower edge of the mediastinal tumor with an ultrasonic knife. The intact thymus gland and its surrounding tissues was divided into four zones (lower right, upper right, upper left, and lower left) by the anterior median line of the sternum and the lower pole of the left innominate vein (Figure 1C). The lower edge of the tumor was first separated. Starting from the right lower pole of the tumor, the right mediastinal pleura was opened from bottom to top to the suprasternal notch to separate the superior vena cava, the right innominate vein, and other important vessels. The left mediastinal pleura was also opened from the 

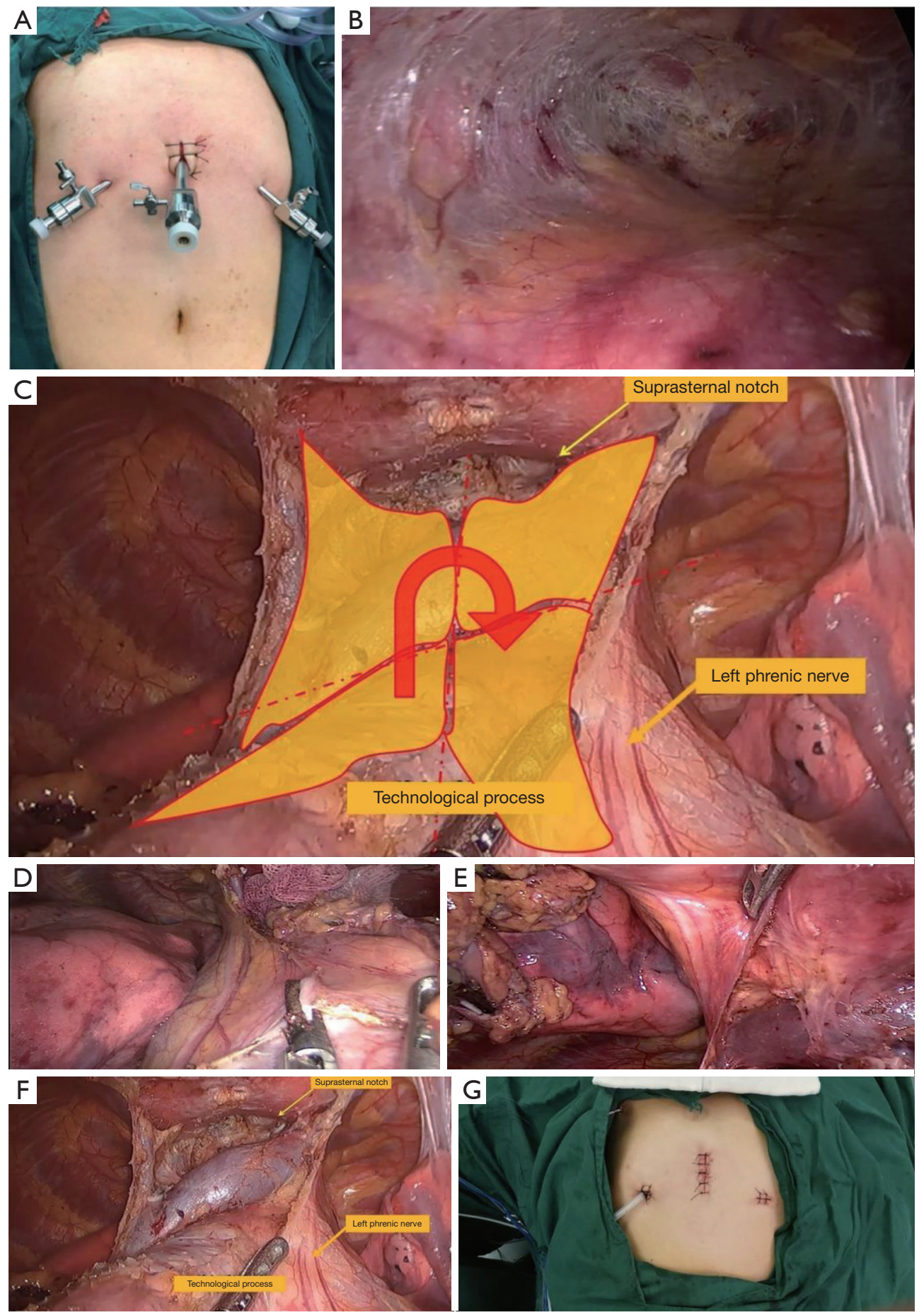

Figure 1 Schematic diagram of the surgical procedures. (A) Incision; (B) posterior sternum interface; (C) schematic diagram of the "four-zone one-way" method; (D) right phrenic nerve surface; (E) left phrenic nerve surface; (F) tumor removal; (G) postoperative incision display. 
left upper pole of the tumor to separate the left innominate vein, left common carotid artery, and left subclavian artery, successively. During the operation, attention was focused on protecting the bilateral phrenic nerve (the left and right boundary of the tumor) (Figure 1D,1E), and finally the upper pole of the thymus tumor was separated. Similar to the traditional method, the scope of dissection of the peri thymus adipose tissue included the bilateral anterior mediastinum, the diaphragmatic angle, and the peri thymus adipose tissue (Figure $1 F$ ). The fat was removed completely, together with the anterior mediastinal tumor, and the specimens were placed into the specimen extractor through the operating hole and dragged out. After complete hemostasis, the wound was covered with hemostasis material and sprayed with biological glue preparation. One posterior sternal drainage tube was indwelling. After gauze and instruments were counted, the incision was sutured layer by layer (Figure 1G).

\section{Assessment of outcomes}

The operative time, intraoperative blood loss, postoperative complications, postoperative hospitalization days, postoperative thoracic drainage volume, days of intrathoracic drainage tube retention, and duration of painkiller use were observed and compared between the two groups. To assess the postoperative effects on MG, the difference in the preoperative and postoperative quantitative $\mathrm{MG}$ scoring system score (QMG-score) and the dose reduction of cholinesterase inhibitors were examined. In particular, QMG-score is a scoring system based on quantitative testing of sentinel muscle groups (Table 1) (10). Cumulative sum analysis (CUSUM) (11) was applied to find the operation time (OT)-learning curve of the S-VATS "four-zone oneway" method. CUSUM analysis detects the deviation between the raw data of each individual case and the mean value of the cohort, followed by the accumulation of each deviation in a sequential manner. All cases with abnormal values were removed.

\section{Data collection and statistical analysis}

Data are presented as mean value \pm standard deviation for continuous variables, and percentages for categorical variables. Student's $t$-test or Mann-Whitney U-test was used to analyze continuous variables, and the Pearson's $\chi^{2}$ test or Fisher's exact test was used to analyze categorical variables using SPSS 21 software .

\section{Results}

\section{Clinical characteristics of the patients}

The clinical characteristics of the included patients are shown in Table 2. Between March 2015 and April 2019, a total of 82 patients were included in this analysis. From March 2015 to October 2015, 40 patients underwent the conventional method of S-VATS. From November 2015, due to improvements in surgical methods, 42 patients underwent the "four-zone one-way" method of S-VATS. There were no statistical differences in age, gender, body mass index (BMI), comorbidities, preoperative $M G$, or pathologic diagnosis between the two groups $(\mathrm{P}>0.05$; Table 2$)$.

\section{Intra- and postoperative outcomes}

As shown in Table 3, patients in the "four-zone one-way" group experienced significantly reduced OTs $(138.50 \pm 29.43$ and $118.00 \pm 28.18$ minutes, respectively; $\mathrm{P}=0.002)$ and intraoperative blood loss $(36.00 \pm 20.16 \mathrm{~mL}$ and $23.92 \pm$ $14.96 \mathrm{~mL}$, respectively; $\mathrm{P}=0.003)$ compared with patients in the conventional group. This demonstrated that the "four-zone one-way" method of S-VATS is advantageous in reducing OT and blood loss.

Postoperative outcomes in the "four-zone one-way" group were similar to the conventional group. There were no significant differences in duration of chest tube, postoperative hospital stays, postoperative thoracic drainage volume, number of days of analgesic use after surgery, postoperative pain score, nor the rate of postoperative complications (Table 3). This suggested that the postoperative safety of "four-zone one-way" method was comparable to the conventional method.

As is showed in Table 4, there were no significant differences in the preoperative and postoperative QMGscores between the treatment groups. In addition, the difference between pre- and postoperative QMG-scores, and the rate of cholinesterase inhibitor dose reduction was comparable between the treatment groups. None of the patients in either treatment group experienced MG crisis. All patients showed improved or stable QMG-scores.

\section{Learning curve}

The OT-learning curves of the surgical team were plotted for the conventional group and the "four-zone one-way" group. According to the CUSUM curve, there were 3 learning curve phases for the operative time (Figure 2). 
Table 1 Q-MG score for disease severity

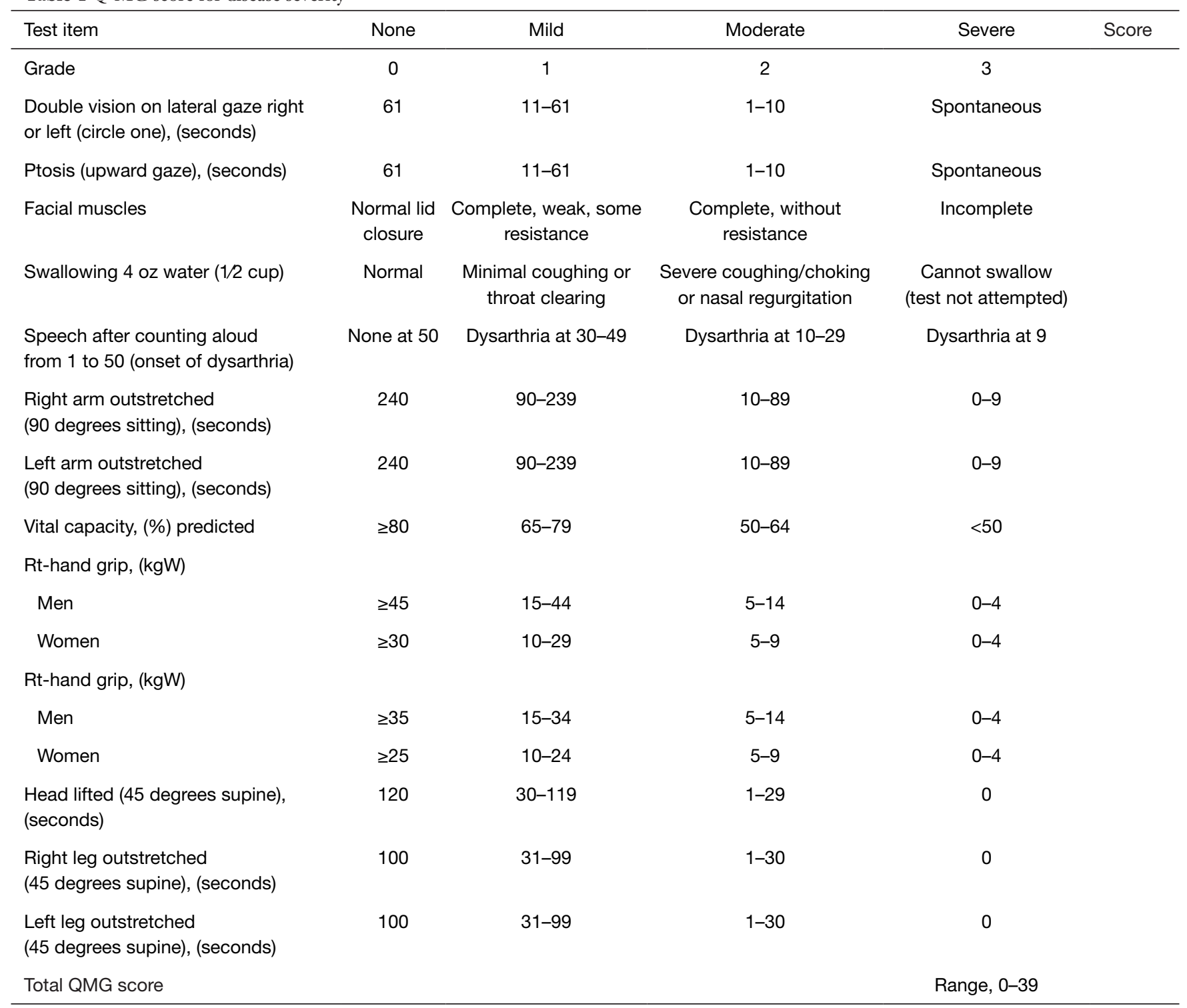

From Barohn RJ, Mclntire D, Herbelin L, et al. Reliability testing of the quantitative myasthenia gravis score. Ann N Y Acad Sci 1998;841:769-72; with permission.

An uphill slope, which is phase I, indicates the period of mastering a surgical method. Figure $2 \mathrm{~A}$ shows that the conventional method can be mastered after 12 cases, while the "four-zone one-way" method can be mastered after only 5 cases (Figure $2 B$ ). There was a "plateau" in phase II which occurred during cases $13-26$ in the conventional method and cases 6-28 in the "four-zone one-way" method. After case 26, the conventional method reached phase III, indicating a period of rapid improvements with mean operative times well below the overall means. In the "four- zone one-way" method, phase III was reached after case 28.

\section{Discussion}

Since the introduction of thoracoscopic surgery for anterior mediastinal tumor resection in 1993, thoracoscopic assisted minimally invasive surgery has become an alternative approach (12). Compared with transthoracic video-assisted thoracic surgery (T-VATS), S-VATS has been shown to not only reduce OT and estimated blood loss, but also 
Table 2 Perioperative clinical characteristics of the patients

\begin{tabular}{|c|c|c|c|c|}
\hline Variable & Total, N (\%) & Conventional method, N (\%) & "Four-zone one-way" method, N (\%) & $P$ value \\
\hline Gender & & & & 0.246 \\
\hline Male & $34(41.5)$ & $14(35.0)$ & $20(47.6)$ & \\
\hline Female & $48(58.5)$ & $26(65.0)$ & $22(52.4)$ & \\
\hline$\leq 25$ & $63(76.8)$ & $32(80.0)$ & $31(73.8)$ & \\
\hline$>25$ & $19(23.2)$ & $8(20.0)$ & $11(26.2)$ & \\
\hline Preoperative complications & & & & 0.391 \\
\hline None & $49(59.8)$ & $22(55.0)$ & $27(64.3)$ & \\
\hline Coronary heart disease & $1(1.2)$ & $1(2.5)$ & $0(0.0)$ & \\
\hline Cases with $M G$ & $41(50.0)$ & $21(52.5)$ & $20(47.6)$ & 0.696 \\
\hline Pathology & & & & 0.900 \\
\hline Thymoma & $20(24.4)$ & $10(25.0)$ & $10(23.7)$ & \\
\hline No thymoma & $62(75.6)$ & $30(75.0)$ & $32(76.2)$ & \\
\hline
\end{tabular}

BMI, body mass index; MG myasthenia gravis.

Table 3 A comparison of intra- and postoperative outcomes

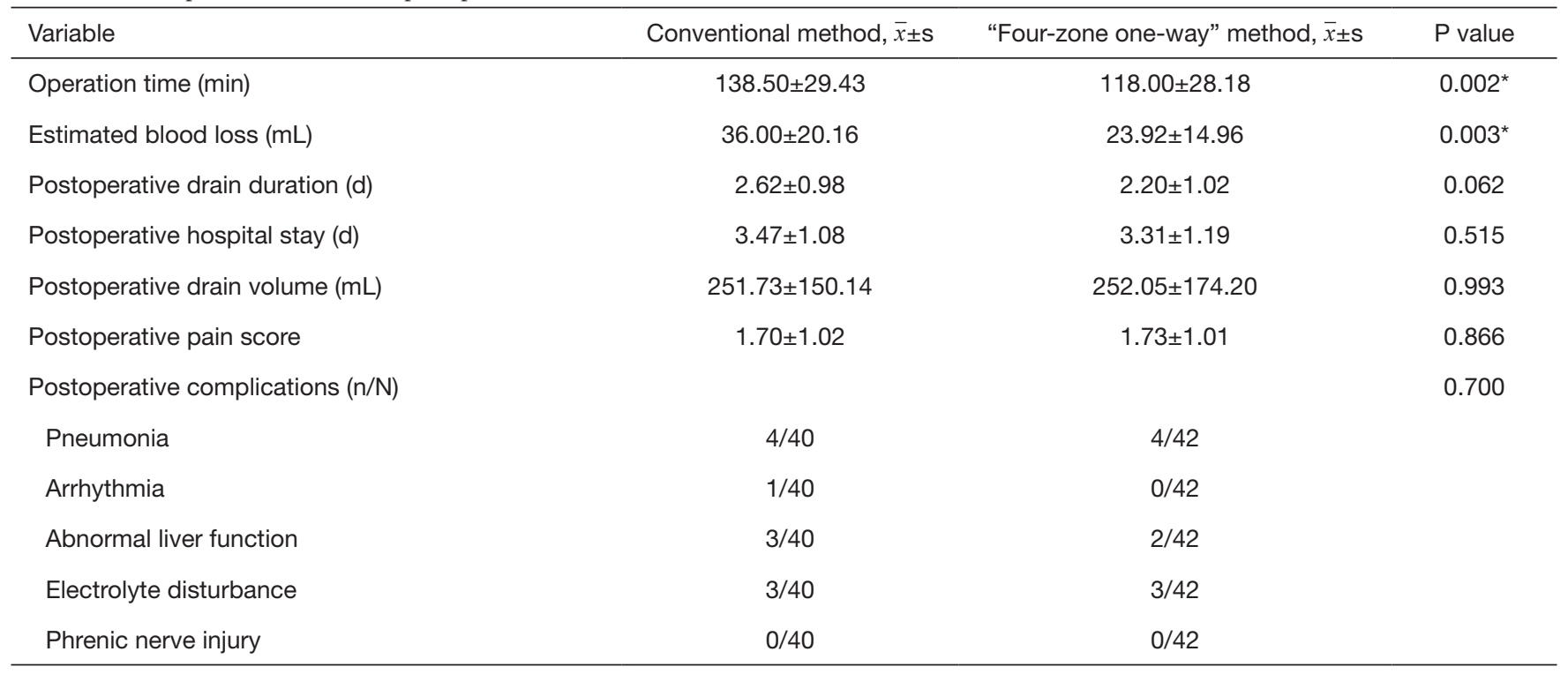

*, $\mathrm{P}<0.05$. 
Table 4 A comparison of the postoperative effects on myasthenia gravis

\begin{tabular}{|c|c|c|c|}
\hline Variable & Conventional method, $\bar{x} \pm \mathrm{s}$ & "Four-zone one-way" method, $\bar{x} \pm \mathrm{s}$ & $P$ value \\
\hline Postoperative QMG-score & $2.48 \pm 1.83$ & $2.55 \pm 1.93$ & 0.901 \\
\hline Change in pre- and postoperative QMG-scores & $1.76 \pm 1.45$ & $1.60 \pm 1.10$ & 0.689 \\
\hline Improved or stable QMG-score & $21(100 \%)$ & $20(100 \%)$ & - \\
\hline MG crisis & 0 & 0 & - \\
\hline
\end{tabular}

QMG, quantitative MG; MG, myasthenia gravis.
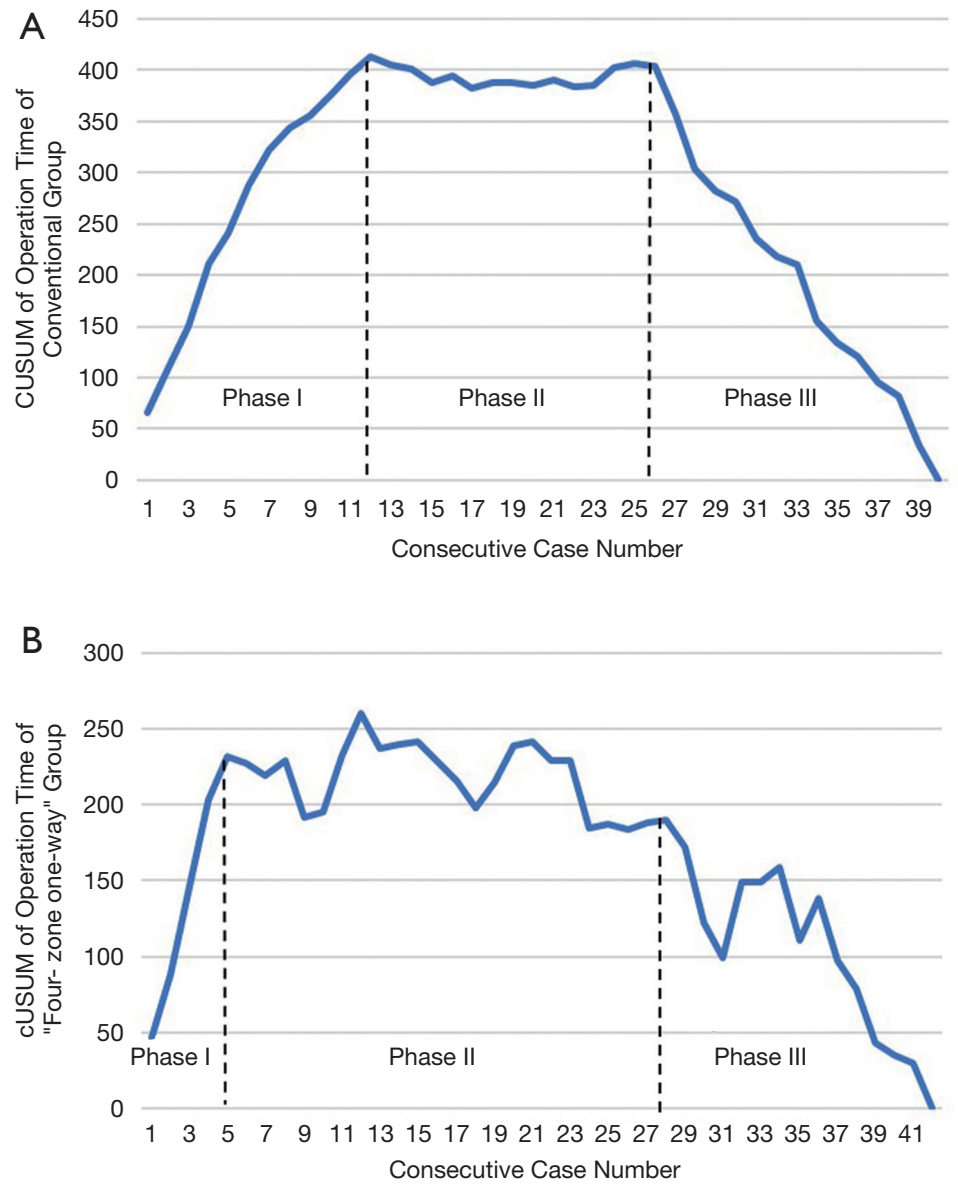

Figure 2 Cumulative sum (CUSUM) analysis of the operation time (OT) of the conventional group and the "four-zone one-way" group. The $\mathrm{X}$ axis indicates consecutive cases, and the $\mathrm{Y}$ axis indicates the cumulative sum of the operative time of each operation and the mean of the operations in the group. The vertical lines at the turning points of the curvature represent the transition from one phase to another.

postoperative pain due to the avoidance of intercostal nerve injury (13). S-VATS provides a good surgical view of the upper pole of the thymus, the adipose tissue in the diaphragmatic angle, and the bilateral phrenic nerves (14).
Approximately $30 \%$ of thymoma patients present with MG, but only $21 \%$ of myasthenia patients have thymoma (15). The difficulty of surgical treatment in MG patients lies in the removal of the adipose tissue at the diaphragmatic angle on 
both sides, and thus, the postoperative effect depends on the thorough removal of the thymus tissue and the surrounding adipose tissue during the operation (16). Therefore, S-VATS is beneficial for the thorough removal of the thymus gland and its surrounding tissues in patients with MG. However, S-VATS also has the following disadvantages: (I) the small operating space (17) limits the flexibility of the instrument and makes it difficult to operate, and thus requires the operator to have rich experience in endoscopic surgery, and the operation should be gentle and flexible; and (II) for large mediastinal tumors or tumor accumulation of surrounding vital organs, median sternal cleavage is more advantageous than subxiphoid cleavage.

Since March 2015, our center has been performing the first resection of an anterior mediastinal tumor by the conventional S-VATS method. From May 2017, on the basis of the conventional S-VATS, the "four-zone one-way" method was pioneered. Compared with the conventional S-VATS, the "four-zone one-way" method has several advantages. First, the surgical area is clearly divided, with the thymus divided into four areas (lower left, upper left, upper right, and lower right) by the anterior median line of the sternum and the horizontal line of the lower pole of the left innominate vein (two lines) during the operation. The surgeon can thus clearly define which structures are to be excised and retained in each area. Second, the surgical method is clear, with the operation starting from the diaphragmatic angle, and the tumor resection performed in a single direction (clockwise or counterclockwise). The operation method is relatively fixed and gradual, from simple to complex, and the surgeons can follow the surgical steps and ensure the operation process is orderly. Meanwhile, injury of important blood vessels and nerves in the anterior mediastina and other tissues can be avoided. Third, this method allows for tacit cooperation between the surgeon and the assistant, thereby improving teamwork and reducing OT. In our study, there were no differences in the preoperative characteristics of age, gender, BMI, comorbidities, preoperative MG, or pathologic diagnosis between patients in the two groups. Patients in the "fourzone one-way" group had significantly reduced OT $(138.50 \pm 29.43$ and $118.00 \pm 28.18$ minutes, respectively; $\mathrm{P}=0.002)$ and blood loss $(36.00 \pm 20.16$ and $23.92 \pm 14.96 \mathrm{~mL}$, respectively; $\mathrm{P}=0.003$ ) compared to patients in the conventional group, suggesting that the "four-zone oneway" method of S-VATS has advantages in reducing OT and blood loss.

There were no significant differences in postoperative complications between the groups. The complication rate was $22.5 \%(9 / 40)$ in the conventional group and $19.0 \%$ $(8 / 42)$ in the "four-zone one-way" group. Among all postoperative complications, pulmonary infections have been shown to be the most common, with an incidence of about $1.5 \%$, while in chest surgery, the incidence of postoperative pulmonary infection is about $13-17.5 \%$ $(18,19)$. In our study cohort, 5 patients in the conventional group experienced major complications, including 4 case of pneumonia and 1 cases of arrhythmia. In the "four-zone one-way" group, there were also 4 cases of pneumonia. The QMG scoring systems and the rate of dose reduction of cholinesterase inhibitors were used to assess patients with MG (9,20). As is showed in Table 4, there were no significant differences in preoperative QMG-scores, postoperative QMG-scores, the difference between preoperative and postoperative QMG-scores, nor the rate of the dose reduction of cholinesterase inhibitors between the 2 treatment groups. In the conventional group, $76.2 \%$ showed a dose reduction of cholinesterase inhibitors compared with $80.0 \%$ in the "four-zone one-way" group. All patients in both groups showed improved or stable QMG-score. There were no cases of crisis in our study cohort. This data indicated that there was no difference in the efficacy of treatment of MG between the 2 groups. Further studies are required to determine the long-term remission rate of $M G$.

OT-learning curves of the surgical team were plotted for the conventional method and "four-zone one-way" method. According to the CUSUM curve, there were 3 learning curve phases for the operative time (Figure 2). There was a steady improvement over phase I (cases 1-12 and cases 1-5 for the conventional and "four-zone one-way" methods, respectively), which shows the initial proficiency. This was followed by a "plateau" in phase II (cases 13- 26 and cases 6-28 for the conventional and "four-zone one-way" methods, respectively). Phase III was reached after case 26 in the conventional group and case 28 in the "four-zone one-way" group. In this latter phase, improvements were more rapid, with mean operative times well below the overall means.

This study was performed at a single institution with a small sample size and this may have led to potential bias. Thymoma is a type of anterior mediastinal tumor with low malignancy. After active treatment, satisfactory shortterm results have been obtained. As recommended by the guidelines, longer follow-up times are warranted in the future to further determine the efficacy of the subxiphoid approach. The feasibility and safety of the "four-zone 
one-way" method of S-VATS was comparable to the conventional method of S-VATS.

\section{Acknowledgments}

Funding: The Fujian Young Teacher Fund (grant Nos. JAT170230 and JAT180182), Startup Fund for Scientific Research at Fujian Medical University (grant Nos. 2019QH1031).

\section{Footnote}

Reporting Checklist: The authors have completed the STROBE reporting checklist. Available at https://dx.doi. org/10.21037/atm-21-4070

Data Sharing Statement: Available at https://dx.doi. org/10.21037/atm-21-4070

Conflicts of Interest: All authors have completed the ICMJE uniform disclosure form (available at https://dx.doi. org/10.21037/atm-21-4070). The authors have no conflicts of interest to declare.

Ethical Statement: The authors are accountable for all aspects of the work in ensuring that questions related to the accuracy or integrity of any part of the work are appropriately investigated and resolved. All procedures performed in this study involving human participants were in accordance with the Declaration of Helsinki (as revised in 2013). The study was approved by institutional ethics board of Fujian Medical University [No.: 2021 Association Ethics Examination No. (030)] and informed consent was taken from all the patients.

Open Access Statement: This is an Open Access article distributed in accordance with the Creative Commons Attribution-NonCommercial-NoDerivs 4.0 International License (CC BY-NC-ND 4.0), which permits the noncommercial replication and distribution of the article with the strict proviso that no changes or edits are made and the original work is properly cited (including links to both the formal publication through the relevant DOI and the license). See: https://creativecommons.org/licenses/by-nc-nd/4.0/.

\section{References}

1. Bacha EA, Chapelier AR, Macchiarini P, et al. Surgery for Invasive Primary Mediastinal Tumors. The Society of Thoracic Surgeons 1998:6.

2. Friedant AJ, Handorf EA, Su S, et al. Minimally Invasive versus Open Thymectomy for Thymic Malignancies: Systematic Review and Meta-Analysis. J Thorac Oncol 2016;11:30-8.

3. Odaka M, Shibasaki T, Kato D, et al. Comparison of oncological results for early- and advanced-stage thymomas: thoracoscopic thymectomy versus open thymectomy. Surg Endosc 2017;31:734-42.

4. Kido T, Hazama K, Inoue $Y$, et al. Resection of anterior mediastinal masses through an infrasternal approach. Ann Thorac Surg 1999;67:263-5.

5. Marulli G, Rea F, Melfi F, et al. Robot-aided thoracoscopic thymectomy for early-stage thymoma: a multicenter European study. J Thorac Cardiovasc Surg 2012;144:1125-30.

6. Numanami H, Yano M, Yamaji M, et al. Thoracoscopic Thymectomy Using a Subxiphoid Approach for Anterior Mediastinal Tumors. Ann Thorac Cardiovasc Surg 2018;24:65-72.

7. Zhang L, Li M, Jiang F, et al. Subxiphoid versus lateral intercostal approaches thoracoscopic thymectomy for non-myasthenic early-stage thymoma: A propensity score -matched analysis. Int J Surg 2019;67:13-7.

8. Mao Y, Lan Y, Cui F, et al. Comparison of different surgical approaches for anterior mediastinal tumor. J Thorac Dis 2020;12:5430-9.

9. Xu H, Liu D, Li Y, et al. The Outcomes of Subxiphoid Thoracoscopic Versus Video-Assisted Thoracic Surgery for Thymic Diseases. J Laparoendosc Adv Surg Tech A 2020;30:508-13.

10. Barohn RJ, Mcintire D, Herbelin L, et al. Reliability testing of the quantitative myasthenia gravis score. Ann NY Acad Sci 1998;841:769-72.

11. Biau DJ, Porcher R. A method for monitoring a process from an out of control to an in control state: Application to the learning curve. Stat Med 2010;29:1900-9.

12. Sugarbaker DJ. Thoracoscopy in the management of anterior mediastinal masses. Ann Thorac Surg 1993;56:653-6.

13. Zielinski M, Czajkowski W, Gwozdz P, et al. Resection of thymomas with use of the new minimally-invasive technique of extended thymectomy performed through the subxiphoid-right video-thoracoscopic approach with double elevation of the sternum. Eur J Cardiothorac Surg 2013;44:e113-9; discussion e119.

14. Suda T. Single-port thymectomy using a subxiphoid approach-surgical technique. Ann Cardiothorac Surg 
2016;5:56-8.

15. Raza A, Woo E. Video-assisted thoracoscopic surgery versus sternotomy in thymectomy for thymoma and myasthenia gravis. Ann Cardiothorac Surg 2016;5:33-7.

16. Kondo K, Monden Y. Therapy for thymic epithelial tumors: a clinical study of 1,320 patients from Japan. Ann Thorac Surg 2003;76:878-84; discussion 884-5.

17. Liu CC, Wang BY, Shih CS, et al. Subxiphoid singleincision thoracoscopic left upper lobectomy. J Thorac Cardiovasc Surg 2014;148:3250-1.

18. Lugg ST, Agostini PJ, Tikka T, et al. Long-term impact of developing a postoperative pulmonary complication after lung surgery. Thorax 2016;71:171-6.

19. Arozullah AM, Khuri SF, Henderson WG, et al. Development and validation of a multifactorial risk index for predicting postoperative pneumonia after major noncardiac surgery. Ann Intern Med 2001;135:847-57.

20. Jaretzki A 3rd, Barohn RJ, Ernstoff RM, et al. Myasthenia gravis: recommendations for clinical research standards. Task Force of the Medical Scientific Advisory Board of the Myasthenia Gravis Foundation of America. Ann Thorac Surg 2000;70:327-34.

(English Language Editor: J. Teoh)

Cite this article as: Gao L, Lu J, Shen Z, Chen H, Kang M. A novel method of subxiphoid video-assisted thoracic surgery for thymectomy. Ann Transl Med 2021;9(16):1339. doi: 10.21037/ atm-21-4070 